\title{
Failed Reticulocyte Count in a Patient after Cardiac Surgery
}

\author{
Norbert Ostendorf, $M D^{1^{*}}$ (D) and Veronika Jennissen, $M D^{2}$ (iD \\ ${ }^{1}$ Institute for Laboratory Medicine and Transfusion Medicine, St. Franziskus Hospital Münster, Germany \\ ${ }^{2}$ Institute for Clinical Chemistry, University Hospital of Cologne, Germany
}

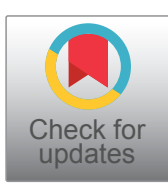

\begin{abstract}
Reticulocyte counting is routinely performed by flow cytometry after applying a fluorescent RNA-stain. We report here a patient with an aberrant population of red blood cells leading to an extremely high and falsely elevated reticulocyte count.
\end{abstract}

\section{Introduction}

The evaluation of erythropoesis by reticulocyte analysis is a mainstay in the diagnosis of anaemia. The classical method comprises staining with brilliant cresyl blue and counting the number of reticulated red blood cells under the microscope. Current hematology analysers perform reticulocyte counting via fluorescence flow cytometry after staining with a dye that adheres to nucleic acid. This has almost completely replaced the manual counting, which is labour intensive. The differentiation between mature erythrocytes and reticulocytes is done automatically by the software. The results of these measurements are rarely questioned, even when they are outright implausible. This case report shows why reticulocyte numbers under certain conditions should be treated with caution.

\section{Case Presentation}

A 78-year-old patient had a cardiopulmonary bypass procedure in a tertiary care hospital and was afterwards transferred to our general hospital for further care. During the operation the patient received 8 units of packed red cells. The full blood count showed a moderate anaemia with haemoglobin concentration of $9.1 \mathrm{~g} / \mathrm{dl}$, erythrocytes 3.15 $\mathrm{G} / \mathrm{l}$ but a remarkable reticulocytosis of $27.3 \%$ or $0.862 \mathrm{G} / \mathrm{l}$. If these figures were correct, the whole population of red cells would be replaced approximately every 4 days. Hematologic investigations were carried out using a Sysmex XN-1000 analyser (Sysmex Germany, Norderstedt).

The data of the reticulocyte measurement are plotted as a scattergram (Figure 1). This normally shows different populations that are clearly distinguishable and are indicated by different colours. The boundaries between such populations are formed automatically by the software according to a proprietary algorithm.

The scattergram of the patient revealed two completely separated populations of red blood cells, as discriminated by fluorescence. This led to a disturbance of the calculations. The margin between the erythrocytes and the lowly fluorescent reticulocytes was drawn through the middle of the second erythrocyte cluster with higher fluorescence (Figure 2), resulting in the implausibly high reticulocyte count. The instrument correctly showed an error flag "RET abnormal scattergram", warning that the measurement was unreliable.

This observation was retrospectively confirmed in the tertiary care hospital on their Sysmex XN-9100. The result of this analysis was even higher with $44.5 \%$ reticulocytes. The erythrocyte count was $3.37 \mathrm{G} / \mathrm{l}$.

The case was discussed on the hotline with the manufacturer. They confirmed that the results were implausible and recommended a manual reticulocyte count instead using brilliant cresyl blue stain and resulted in a value of $1.6 \%$ reticulocytes. The blood smear revealed no morphologic abnormalities of the erythrocytes apart from anisocytosis.

Unfortunately, the reason for the different staining behaviour of these red cell populations is unknown and remains speculative. Two mechanisms come into question: there was a break in the continuity of erythropoiesis, maybe caused by the operative procedure or the anaesthesia [1]. This seems possible if the progression of the cells on the diagram from right to left is regarded as a sign of ageing or maturing.

*Corresponding author: Norbert Ostendorf, MD, Institute for Laboratory Medicine and Transfusion Medicine, St. Franziskus Hospital Münster, Hohenzollernring 70, 48147 Münster, Germany

Accepted: January 10, 2022

Published online: January 12, 2022

Citation: Ostendorf N, Jennissen V (2022) Failed Reticulocyte Count in a Patient after Cardiac Surgery. Clin Hematol Res 5(1):59-61 


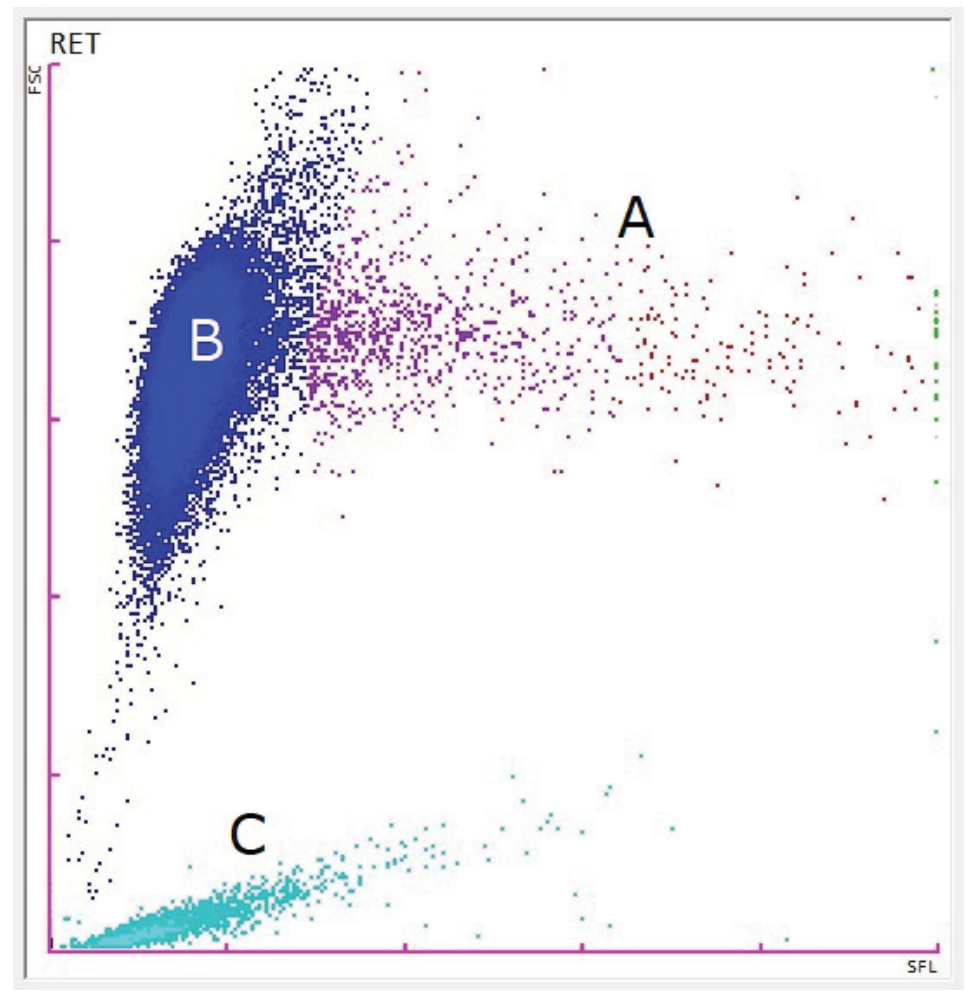

Figure 1: Normal scattergram of a reticulocyte count (Sysmex XN-1000). The side scatter fluorescence (sfl) is plotted on the x-axis, the forward scatter ( $\mathrm{fsc}$ ) on the $y$-axis. The cells are stained with an RNA-stain. The events with high fluorescence are reticulocytes (A). The blue cloud on the left represents mature red blood cells with low fluorescence; (B). The population on the bottom of the diagram shows the platelets; (C). Colourisation is managed by the software.

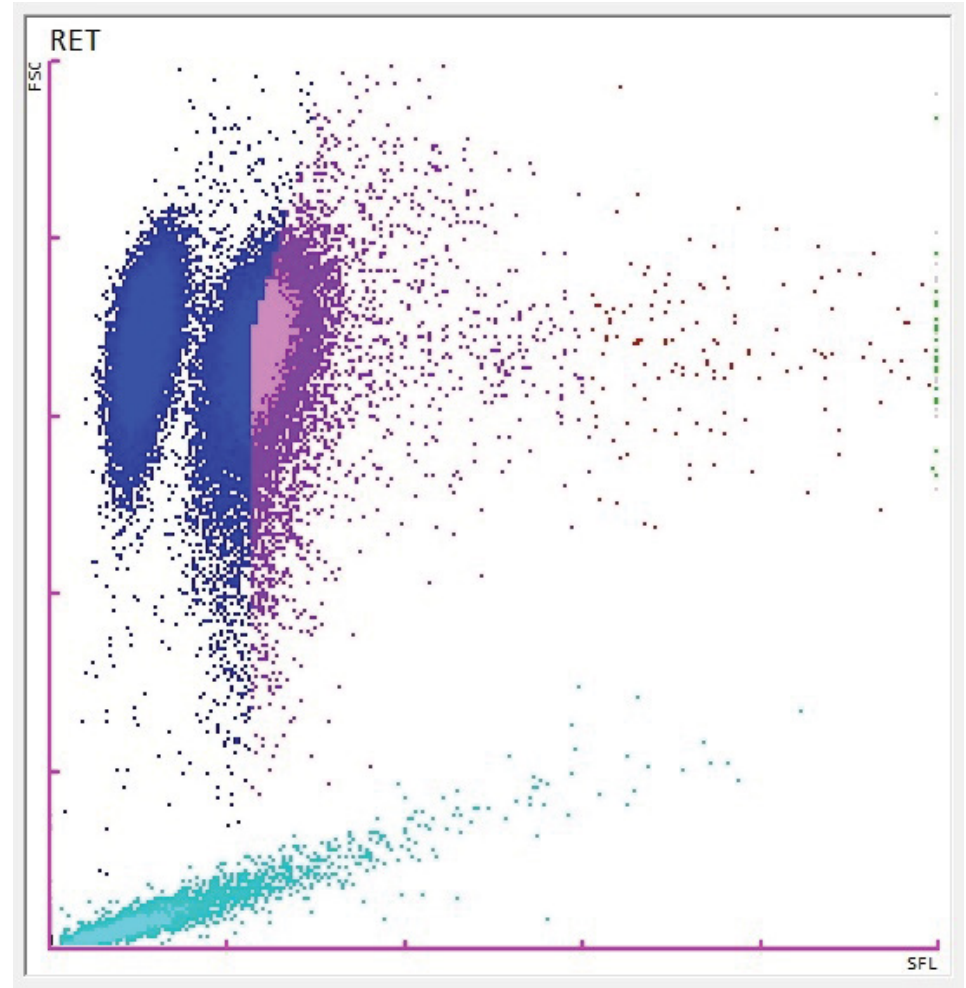

Figure 2: Scattergram of the patient's blood sample showing two distinct populations of red blood cells. The automatic discrimination between mature red cells and reticulocytes obviously runs through the right cluster of red cells, leading to a falsely elevated reticulocyte count. 
On the other hand, the extracorporeal circulation may have damaged the membrane of the erythrocytes, causing a better penetration of the RNA-stain [2].

\section{Discussion}

Clinicians and laboratory specialist alike should be aware that the automatic reticulocyte count is not fail proof. A simple calculation can reveal a possible implausibility. In normal erythropoiesis, reticulocytes amount for slightly under $1 \%$ of the red cells, and the turnover of erythrocytes is 120 days. $10 \%$ reticulocytes are equivalent to a turnover of 12 days and $20 \%$ of 7 days. The shorter this lifespan, the more implausible is the reticulocyte count. This is especially true when the reticulocyte hemoglobin content is very high or low, which is an indicator of impaired erythropoiesis.

\section{Compliance with Ethical Standards}

\section{Conflict of interest}

Norbert Ostendorf has received a speaker honorarium from Sysmex Deutschland GmbH. Veronika Jennissen declares that she has no conflict of interest.

\section{Ethical approval}

All procedures performed in studies involving human participants were in accordance with the ethical standards of the institutional and/or national research committee and with the 1964 Helsinki declaration and its later amendments or comparable ethical standards.

\section{Informed consent}

Informed consent was considered not necessary because this case report was drawn purely from routine data in anonymized form without any risk for the patient or infringement of patient rights.

\section{References}

1. Kumar M, Bhoi S (2016) Impaired hematopoietic progenitor cells in trauma hemorrhagic shock. J Clin Orthop Trauma 7: 282-285.

2. Olia SE, Maul TN, Antaki JF, et al. (2016) Mechanical blood trauma in assisted circulation: Sublethal RBC damage preceding hemolysis. Int J Artif Organs 39: 150-159.

DOI: $10.36959 / 831 / 386$ 